\title{
Thrombophilic screening in retinal artery occlusion patients
}

\author{
Valeria Nagy' \\ Lili Takacs' \\ Zita Steiber' \\ György Pfliegler ${ }^{2}$ \\ Andras Berta' \\ 'Department of Ophthalmology, \\ ${ }^{2}$ Division of Rare Diseases, University \\ of Debrecen Medical and Health \\ Science Center, Debrecen, Hungary
}

Background: Retinal artery occlusion (RAO) is an ischemic vascular damage of the retina which frequently leads to sudden, mostly irreversible loss of vision. In this study, blood thrombophilic factors as well as cardiovascular risk factors were investigated for their relevance to this pathology. Thrombophilic risk factors so far not evaluated were included in the study.

Patients and methods: 28 RAO patients and 81 matched control subjects were examined. From blood samples, protein $\mathrm{C}$, protein $\mathrm{S}$, antithrombinopathy, and factor $\mathrm{V}$ (Leiden) mutation (FV), factor II gene polymorphism, factor VIII C level, plasminogen activity, lipoprotein(a) and fibrinogen levels, hyperhomocysteinemia and presence of anticardiolipin - antiphospholipid antibodies were investigated. Possibly relevant pathologies such as diabetes mellitus, hypertension, and ischemic heart disease were also registered. Statistical analysis by logistic regression was performed with $95 \%$ confidence intervals.

Results: In the group of patients with RAO only the incidence of hypertension (OR: 3.33, 95\% CI: $1.30-9.70, p=0.014$ ) as an average risk factor showed significant difference, but thrombophilic factors such as hyperfibrinogenemia (OR: 2.9, 95\% CI: $1.29-6.57, p=0.010$ ) and the presence of FV (Leiden mutation) (OR: 3.9, 95\% CI: 1.43-10.96, $p=0.008$ ) increased the chances of developing this disease.

Conclusions: Our results support the assumption that thrombophilia may contribute to the development of RAO besides vascular damage due to the presence of cardiovascular risk factors. Further studies are needed, however, to justify the possible use of secondary prophylaxis in form of anticoagulant/antiplatelet therapy.

Keywords: retinal arterial occlusion, risk factors, thrombophilia

\section{Introduction}

Von Graefe (1859) was the first to describe the classic clinical picture of retinal artery occlusion. He observed a man who had experienced a sudden loss of vision in the right eye with the following fundus findings: pale disk, attenuated arteries and veins, and cloudy retina that produced a macular cherry-red spot.

Clinicians usually differentiate acute retinal arterial occlusion (RAO), such as central retinal artery occlusion (CRAO) and branch retinal artery occlusion (BRAO). Fundoscopy characteristics of CRAO patients are the pale, ischemic retinal edema with a cherry red spot in the macula, and arteriolar narrowing or slugging with retinal arterioles irregularities in their caliber (Bertram et al 1995; Ben-Ami et al 2002; Cahill et al 2003; Chua et al 2006).

Fundus changes of BRAO are white, ischemic retina in the area supplied by an obstructed artery. In addition, capillary changes have also been described.

RAO is a multifactorial disorder. Next to the role of cardiovascular risk factors, thrombophilic factors have also been shown to play a role. The exact etiology and pathogenesis for RAO is still not clear (Müller et al 1992; Wenzel et al 1993; Bertram et al 1995; Geigner et al 1999; Pianka et al 2000; Saloman et al 2001; 
Ben-Ami et al 2002; Weger et al 2003; Stojakovic et al 2007). This is one of the reasons why targeted therapy for RAO is not available.

Our study investigated the pathogenetic factors from the widely accepted, so-called "acquired" cardiovascular risk factors (hypertension, hypercholesterolemia) in parallel with thrombophilic risk factors (Müller et al 1992; Geigner et al 1999; Pianka et al 2000). We predict that our knowledge of the pathogenetic risk factors of RAO may enable us to define therapy and secondary prophylaxis as well.

\section{Patients and methods}

Between 1997 and January of 2006, we studied 28 RAO patients and 81 age- and sex-matched control subjects in our Department of Ophthalmology within the Division of Rare Diseases. The mean age was $61.1( \pm 12.3)$ years in the RAO patients and $61.6( \pm 12.6)$ years the control subjects. The inclusion criteria for RAO were: sudden, painless loss of vision, presence of relative afferent pupillary defects, characteristic visual field defects, and fundus changes. The following fundoscopic findings were recorded: pale, ischemic retinal edema with a cherry red spot in the macula, and arteriolar narrowing or slugging with retinal arterioles irregularities in their caliber. Whenever the above changes were detected in all the four arterioles' branches we referred to it as central occlusion (CRAO), and if one arteriole area was seen, the branch occlusion (BRAO) was established.

Exclusion criteria for patients with RAO comprised malignancy and vasculitis. To exclude vasculitis, the erythrocyte sedimentation rate (ESR) and C-reactive protein (CRP) levels were determined. The possibility of vasculitis was considered when the ESR level was higher than $33 \mathrm{~mm} / \mathrm{h}$ and $35 \mathrm{~mm} / \mathrm{h}$ in men and women, respectively, or when the CRP was higher than $0.5 \mathrm{mmol} / \mathrm{l}$. We examined the presence of general symptoms such as headache, muscle ache, other, etc.

The control group consisted of 81 age- and sex-matched patients who have previously undergone cataract surgery, and subsequently presented retinal vascular diseases. In this case, at least one year had passed between the cataract surgery and the follow-up examination. No patients had suffered from autoimmune diseases. All patients were given detailed description of the main points of the examination, to which they then gave their written consent. We registered the previous diseases and medical therapy of our patients prior to their first ophthalmologic examination. Those patients who were considered hypertensive, in that an internal medicine specialist had diagnosed them based on a measurement of higher than 140/90 $\mathrm{mmHg}$ on several occasions, also received scheduled controls as well as antihypertensive therapy. Patients suffering from hypercholesterolemia (above $5.2 \mathrm{mmol} / \mathrm{l}$ ) or hypertriglyceridemia (above $1.2 \mathrm{mmol} / \mathrm{l}$ ) took part in oral antihyperlipidemic therapy.

All the patients control groups were subjected to a complete ophthalmologic examination, including best corrected visual acuity, a visual field test using Goldmann perimetry when visual acuity allowed it, intraocular pressure measurement using Goldmann applanation tonometry, slit lamp biomicroscopy and fundoscopy, as well as fluorescein angiography.

The risk factors (hypertension, diabetes mellitus, ischemic heart disease, arteriosclerosis, and smoking) of each patient were also documented. In addition to the routine laboratory investigations, the level of serum cholesterol and triglyceride were measured, and an hemostasis screening test (prothrombin time, thrombin time, activated partial thromboplastin time) was performed. The starting date of the first ophthalmologic symptoms was noted and six weeks later the blood samples were taken to evaluate thrombophilic risk factors: protein C (PC) and protein S (PS) levels, antithrombinopathy (AT), activated protein-C (APC) resistance and Leiden mutation, presence of FII G20210A allele, fibrinogen level, anticardiolipin - antiphospholipid (ACL-APL) antibodies, elevated levels of factor VIII (FVIII), plasminogen, and lipoprotein-a (Lp(a)). The level of FVIII was considered elevated only when it increased in three consecutive measurements, made at 6-week intervals after the initial screening. This way, we could filter out the false positive results. Since FVIII also plays a role as an acute phase protein, elevated levels can be found in the acute RAO phase without actually having any pathological role. We have followed the same protocol for the determination of decreased levels of PC, PS, and AT.

Control examinations were dated 6 weeks, 3 to 6 months, and one year after the initial examination. The total follow up time was between 11 months to 9 years. Nine patients were followed over a 9 year period.

\section{Laboratory methods}

All laboratory analysis was performed at the Department of Clinical Biochemistry and Molecular Pathology, University of Debrecen Medical and Health Science Center, Debrecen, Hungary.

Determinations of lipid (triglyceride, cholesterol) levels were performed using the enzymatic colorimetric method 
in a P800 modular chemical analyzer (Roche Diagnostics, Mannheim, Germany). Fibrinogen levels were determined according to the Clauss method (Clauss 1957). PC and PS activities were measured using coagulation tests (Diagnostica Stago). AT and plasminogen were determined by chromogenic assays (Diagnostica Stago). FVIII was determined using a clotting assay. All these determinations were performed in an STA-Compact coagulometer (Diagnostica Stago). FV Leiden mutation and FII G20210A allele were detected using a real-time polymerase chain reaction (RTPCR) method according to Bertram and colleagues (1995).

Determination of the activated protein-C (APC-R) ratio was achieved by the measurement of two different activated partial thromboplastin time (APTT). One measurement represented the plasma with $\mathrm{APC}$, the other one without APC. The APC ratio is the ratio between these two measurements, which we have considered positive at the level of 2 or higher. In positive cases, the existence of the Leidenmutation is always justified by molecular genetic methods. The potentially abnormal DNA sequences were amplified by polymerase chain reaction (PCR). The PCR products were digested with restriction endonuclease (MnII), and the restriction fragments were separated by agars gel electrophoresis. PCR method was used for the identification of the FII G20210A allele. An enzyme-linked immunosorbent assay (Selisa, Cambridge Life Sciences, UK) was performed to determine the antibody against ACL. Lp(a) concentration was measured using a chemical method in an Integra 700 automat (Roche Diagnostics). Homocysteine levels were determined by combined enzymatic and fluorescent polarization immune method. In this procedure, all the homocysteine content of the samples is first converted to S-adenosyl homocysteine ( $\mathrm{SAH})$. This product then competes with a manufacturer labeled SAH analogue for antibody binding. The amount of fluorescent analogue was measured by polarizing immune method.

\section{Statistical analysis}

The homogeneous distribution of age and gender among RAO patients and control subjects was confirmed by the Mann-Whitney test and chi-squared test respectively. Patients and control subjects were compared using Student's $t$-test for parameters showing normal distribution, or the MannWhitney test when the parameters' distribution was not normal $(p 1=0.71, p 2=0.11)$.

The thrombophilic and cardiovascular risk factors were analyzed by logistic regression odds ratios with $95 \%$ confidence intervals. $p$-values lower than 0.05 were considered significant. The statistical analysis was performed with SPSS version 11.5 (SPSS Inc., Chicago, IL).

\section{Results}

The mean age of the 16 male and 12 female patients with RAO was $61.12( \pm 12.3)$ years, while that of the control group was $61.6( \pm 12.6)$ years, with a male/female ratio of 53/28 (Table 1).

CRAO was seen in 20 patients, and BRAO was developed in 9 patients. In the RAO patients, logistic regression

Table I Statistical data of retinal artery occlusion patients in cardiovascular and thrombophilic risk factors

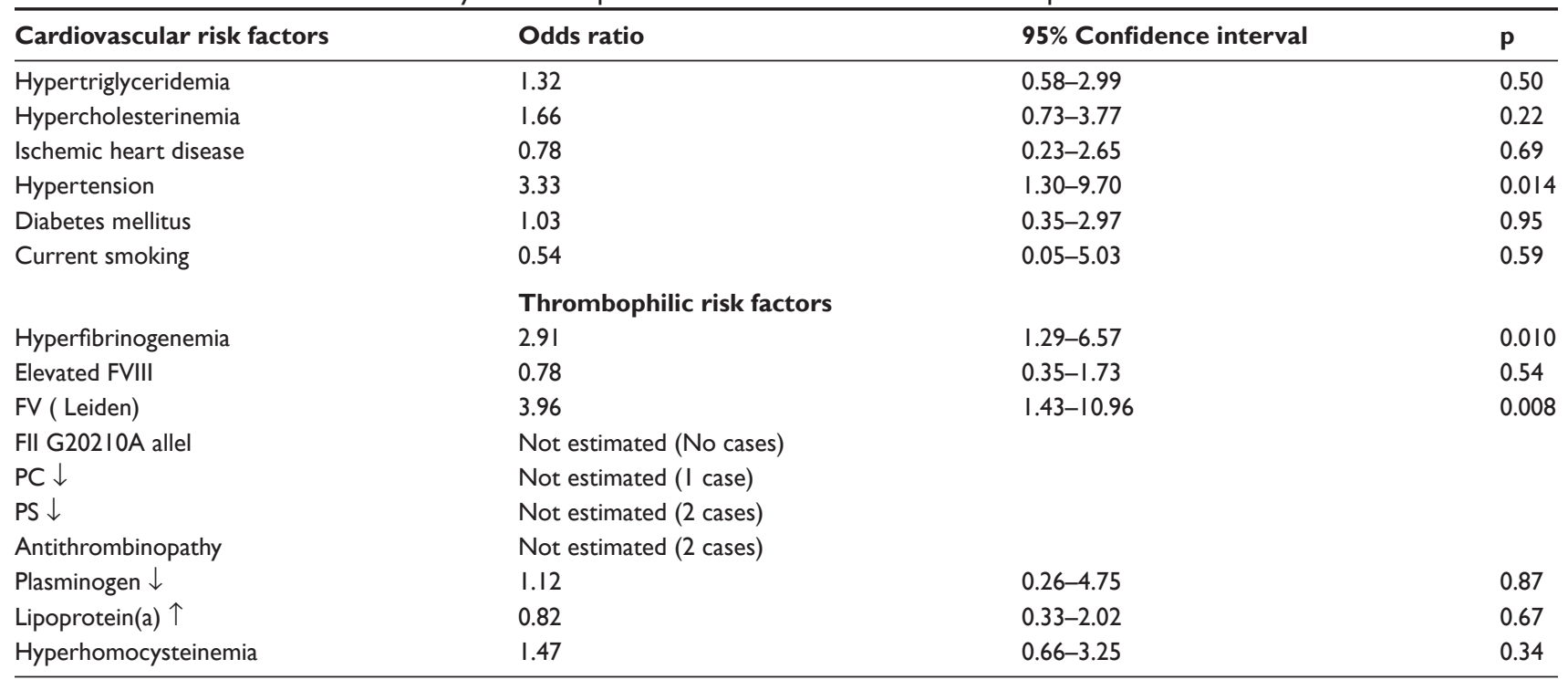

Abbreviations: FVIII, factor VIII FV(Leiden), factor V of Leiden mutation; FII G202IOA, factor II gene polimorphism, G202I0A allele; PC $\downarrow$, decreased level of proteine C; PS $\downarrow$, decreased level of proteine S; plasminogen $\downarrow$, decreased level of plasminogen; Lipoprotein(a) $\uparrow$, increased level of lipoprotein(a). 
odds ratios with $95 \%$ confidence interval were compared for cardiovascular risk factor. Hypertension (OR: 3.33, 95\% CI: $1.30-9.70, p=0.014)$, as an average risk factor, showed significant difference, but thrombophilic risk factors such as hyperfibrinogenemia (OR: 2.9, 95\% CI: 1.29-6.57, $p=0.010$ ) and the presence of APC-resistance FV (Leiden mutation) (OR: 3.9, 95\% CI: 1.43-10.96, $p=0.008$ ) increased the chances of developing this disease.

Statistical analysis could not be performed for other thrombophilic factors such as decrease in PC and PS activities, presence of FII 20210A allele and increase in FVIII, as thrombophilic changes could be observed in only 5 cases of those affected, and none in the control patients. In one case PC was observed, while a PS decrease was demonstrated in 2 cases, and AT were shown in 2 other patients. No patient demonstrated any difference in the F II G20210A allele (Table 2).

Bilateral cases had not developed during the follow-up period.

\section{Discussion}

The multifactorial nature of RAO has been well documented. It may be caused by embolization from the internal carotid artery (thromboemboli or cholesterol emboli originating in atherosclerotic plaques of the carotid arteries), but rarely from the heart (eg, atrial fibrillation, aortic or mitral valve disease, endocarditis and atrial myxoma) (Müller et al 1992; Richard et al 1996; Rumelt et al 1999). The other important risk factors of RAO pathogenesis are cardiovascular risk factors such as hypertension, aortic diseases, diabetes mellitus, atherothrombosis, and current smoking.
Coagulation abnormalities in can play a role its development (Müller et al 1992; Richard et al 1996; Rumelt et al 1999; Pianka et al 2000; Salomon et al 2001; Stojakovic 2007). Increased coagulation may cause ischemic retinal infarction by decreased blood flow of the retinal arterioles. In some cases RAO cannot be explained with the known factors alone thus identification of other unknown risk factors such as thrombophilic risk factors should be considered (Ben-Ami et al 2002).

Weger and colleagues (2003) and Pianka and colleagues (2000) stated that increased homocysteine level plays a role in the development of RAO (Wenzel et al 1993; Cahill et al 2003; Chua et al 2006). In our patients who suffered from RAO, we frequently found such a significant increase. No correlation was found in the methyltetrahydrofolate reductase gene polymorphism between the patients and the controls.

Müller and colleagues (1992) proved that the increased Lp(a) level has a pathological role in the development of RAO. Elevated Lp(a) level can cause arteriosclerotic changes in small arterioles in the carotid system on one hand, and on the other hand, it is considered as a thrombophilic factor due to its antifibrinolytic effect. $\mathrm{Lp}(\mathrm{a})$ binding to plasminogen receptors in the blood vessel endothelium competitively inhibits plaminogen-plasmin transformation. High Lp(a) levels and decreased plasminogen levels have synergistic effects in further increasing thrombophilic risk when both are present at the same time (Müller et al 1992; Stojakovic et al 2007). Plasma Lp(a) levels are high in patients with central retinal artery occlusion. Elevated lipoprotein-a serum level is an established risk factor in cardiovascular and cerebrovascular

Table 2 Statistical data of RAO patients by Mann-Whitney test

\begin{tabular}{|c|c|c|c|}
\hline $\begin{array}{l}\text { Cardiovascular risk factors } \\
\text { (normal level) }\end{array}$ & Mean value & Standard deviation & $\begin{array}{l}\text { Minimum and } \\
\text { maximal value }\end{array}$ \\
\hline Hypertriglyceridemia ( 1.2 mmol/l) & 1.55 & \pm 0.68 & $0.77-3.53$ \\
\hline Hypercholesterolemia (5.2 mmol/l) & 5.92 & \pm 1.23 & $4.17-8.29$ \\
\hline \multicolumn{4}{|l|}{ Thrombophilic risk factors } \\
\hline Fibrinogen level $(>4.0 \mathrm{~g} / \mathrm{l})$ & 4.29 & \pm 1.66 & $2.25-10.56$ \\
\hline FVIII level $(>150 \%)$ & $191.03 \%$ & \pm 57.28 & $126-312$ \\
\hline FV (Leiden) mutation (number of heterozygote) & 4 & & \\
\hline FII G202I0A allel & No case & & \\
\hline PC level (>70\%) & (I case) I37.28 & \pm 29.93 & $67-193$ \\
\hline PS level (>65\%) & ( 2 cases) 108.35 & \pm 20.24 & $54-144$ \\
\hline AT level (>80\%) & ( 2 cases) 114.03 & \pm 11.28 & $76-127$ \\
\hline Plasminogen level (>80\%) & 105.38 & \pm 15.23 & $70-221$ \\
\hline Lp(a) level (>300 mg/l) & 385.24 & \pm 367.2 & $102-1325$ \\
\hline Homocystein level (I $2.5 \mu \mathrm{mol} / \mathrm{I})$ & $|5.4|$ & \pm 12.0 & $10.67-39.43$ \\
\hline
\end{tabular}

Abbreviations: FVIII, factor VIII; FV(Leiden), factor V of Leiden mutation; FII G202IOA, factor II gene polimorphism, G202I0A allele; PC $\downarrow$, decreased level of proteine C; PS $\downarrow$, decreased level of proteine S; plasminogen $\downarrow$, decreased level of plasminogen; Lipoprotein(a) $\uparrow$, increased level of lipoprotein(a). 
diseases. It may have a role in damaging the circulation of the retinal arteries, which may lead to retinal infarction (Müller et al 1992; Stojakovic et al 2007).

Ben-Ami and colleagues (2002) reported showed such case in a 19 year-old-male patient who, despite his young age, developed CRAO in one eye. The patient suffered from no cardiovascular disease and no risk factors could be shown. During the thrombophilic screening, this patient had a homozygous FV Leiden mutation and heterozygous prothombin G20210A polymorphism (Ben-Ami et al 2002).

In the present study, we examined the importance of thrombophilic risk factors in the development of retinal artery occlusion. Our study found that the CRAO-patients have significantly in higher number a FV Leiden mutation, elevated homocysteine level and Lp(a), and our data shows that both cardiovascular and thrombophilic risk factors may have an important role in the development of RAO. Based on our data, we suggest that thrombophilia with or without the presence of vascular damage may have a key role in the development of RAO.

In the case of thrombophilia, causal therapy is also available. It has been proven that cessation of smoking, in conjunction with the administration of vitamins B6, B12, and folic acid, is beneficial in the treatment of homocysteinemia (Wenzel et al 1993; Pianka et al 2000; Cahill et al 2003; Chua et al 2006). When dealing with acute fundus vascular pathology CRAO, the introduction of fibrinolytic therapy is highly recommended (Richard et al 1996; Rumelt et al 1999). Unless otherwise contraindicated, the introduction of thrombolytic therapy should be introduced within 6 hours after the onset of thrombosis. The thrombolytic therapy requires emergency room setting and professional staff. Due to the danger of heavy bleeding side effects, this treatment should not be expanded to a wider circle. It is important to find another effective therapy with lesser side effects.

In the event that thrombophilia has been proved as existing alongside cardiovascular risk factors, we recommend antithrombotic therapy implemented together with the circulation-improving therapy. Antithrombocyte - antiplatelet therapy with salicylates may prove effective, or, if contraindicated, antiplatelet therapy per os is recommended. With this kind of therapy, the likelihood of the development of another arterial event in the other eye decreases.
Thrombophilic screening is recommended for RAO patients under the age of 50, or with bilateral fundus vascular pathology. We recommended thrombophilic screening to other family members if RAO has occurred in the family and the patient has at least 2 strong thrombophilic factors: FV Leiden mutation, PS- or PC-decrease, etc. If cardiovascular risk factors are also present alongside thrombophilic risk factors, then chance of developing RAO gets multiplied. Positive family anamnesis after thrombophilia in selected cases raises a possibility for primary prevention with antithrombotic/ antiplatelet therapy.

\section{References}

Ben-Ami R, Zeltser I, Leibowitz I, et al. 2002. Retinal artery occlusion in a patient with factor V Leiden and prothrombin G20210A mutations. Blood Coagul Fibrinolysis, 13:57-9.

Bertram B, Remky A, Arend O, et al. 1995. Protein C, protein S, and antithrombin III in acute ocular occlusive diseases. German $J$ Ophthalmol, 4:332-5.

Cahill MT, Stinnett SS, Fecrat S. 2003. Meta - analysis of plasma homocysteine, serum folate, serum $B_{12}$, and thermolabile MTHFR genotype as risk factors for retinal vascular occlusive disease. Am J Ophthalmol, 136:1136-50

Chua B, Kifley A, Wong TY, et al. 2006. Homocysteine and retinal emboli: The Blue Mountains eye study. Am J Ophthalmol, 142:322-4.

Clauss A. 1957. Gerinnungsphysiologische schnell Methode zur Bestimmung des Fibrinogens. Acta Hematol, 17:237.

Geigner K, Hafner G, Dick B, et al. 1999. Retinal vascular occlusion and deficiencies in the protein C pathway. Am J of Ophthalmol, 128:69-74.

Müller HM, Diekstall FF, Schmidt E, et al. 1992. Lipoprotein (a): a risk factor for retinal vascular occlusion. German J Ophthalmol, 1:338-41.

Pianka P, Almong Y, Man O, et al. 2000. Hyperhomocysteinemia in patients with nonarteritic anterior ischemic optic neuropathy, central retinal artery occlusion, and central retinal vein occlusion. Ophthalmol, 107:1588-92.

Richard G, Lerche RC, Knospe V, et al. 1996. Treatment of retinal arterial occlusion with local fibrinolysis using recombinant tissue plasminogen activator. Ophthalmol, 106:768-73.

Rumelt S, Dorenboim Y, Rehany U. 1999. Aggressive systemic treatment for central retinal artery occlusion. Am J Ophthalmol, 128:733-8.

Salomon O, Huna-Baron R, Moisseiev J, et al. 2001. Thrombophilia as a cause for central and branch retinal artery occlusion in patients without an apparent embolic source. Eye, 15:511-14.

Stojakovic T, Scharnagl H, Marz W, et al. 2007. Low density lipoprotein triglycerides and lipoprotein(a) are risk factors for retinal vascular occlusion. Clin Chim Acta, 382:77-82.

Von Graefe A. 1859. Ueber Embolie der Arteria centralis retinae also Ursache plotzlichererblindung. Graefe's Arch Ophthalmol, 5:136.

Weger M, Renner W, Pinter O, et al. 2003. Role of factor V Leiden and prothrombin $20210 \mathrm{~A}$ in patients with retinal artery occlusion. Eye, 17: 731-4.

Wenzel EM, Rademakers AJ, Boers GH, et al. 1993. Hyperhomocysteinemia in retinal artery and retinal vein occlusion. Am J Ophthalmol, $115: 162-7$. 
